\title{
DE LA VEDETTE DU SPORT À LA LÉGENDE DU SPORT : LE CAS ANQUETIL
}

\author{
Jean MAURICE ${ }^{1}$
}

Les expressions « vedette du sport » et « légende du sport » sont données hâtivement pour quasi synonymes, alors qu'elles appartiennent en toute rigueur à deux couples de régimes différents, l'opsis et le logos, d'une part, la communication et la littérature, d'autre part. Le passage du statut de " vedette » à celui de « légende » s'effectue en plusieurs étapes : articles de presse éphémères, ouvrages grand public centrés sur le sport, œuvres littéraires. Le cas de la transformation d'Anquetil, vedette controversée à l'image trouble, en légende épurée et déréalisée est un exemple probant de cette métamorphose. On le montre en s'appuyant sur l'exemple du « récit » écrit par Paul Fournel, Anquetil tout seul. Paris : Editions du Seuil, 2012.

Le contenu de cet article surprendra peut-être, parce qu'il fait la part belle à la littérature, a priori inattendue sur le terrain où évoluent les vedettes du sport. Je voudrais pourtant montrer que sa présence, bien qu'un peu décalée, n'y est pas totalement incongrue, en tant qu'elle permet de réévaluer la notion de "légende du sport ", en fait plus rare, plus singulière et plus chargée de sens qu'il n'y paraît.

Dans la langue sportive courante, « légende » n'est pas senti comme fondamentalement différent $\mathrm{d} \mathrm{e}$ « vedette ». Q uand les

1 Jean Maurice est professeur émérite de l'Université de Rouen Normandie.

Recherches en communication, ${ }^{\circ} 50$ - Article publié le 07/10/2019 
deux mots ne sont pas simplement quasi synonymes, au mieux, le premier est employé comme l'hyperbole du second : suite à une grande performance, un champion entre vite "dans la légende » à la Une des journaux... pour en fait vite retomber dans l'oubli. On aurait pourtant tort de s'arrêter à cet abus de langage, car la notion de « légende du sport » reste valide, à condition toutefois d'assumer la solution de continuité entre elle et la vedette.

À cette fin, mon propos ne sera pas de m'interroger, en tant que tel, sur le vedettariat sportif et sur les raisons des exagérations sémantiques auxquelles il donne lieu, mais de me demander comment un champion en son temps célébré par les médias quitte leur sphère, change de statut en étant intégré à l'espace littéraire et ainsi se transfigure en légende, entendue au sens propre du terme.

Je commencerai par indiquer les étapes de cette métamorphose, résultat d'une mise en discours qui dépasse la seule communication. Puis je testerai mon hypothèse en examinant un cas concret, celui de Jacques Anquetil, figure controversée tant qu'on en reste au niveau de l'information ou de l'argumentation, fussent-elles bienveillantes, mais métamorphosée par la littérature en une légende débarrassée de ses scories humaines.

\section{De la vedette à la légende, de la communication à la littérature}

Le terme « vedette », dont l'étymon est le verbe latin vedere (voir), renvoie d'emblée au monde du spectacle. Dans le premier tiers du $\mathrm{XIX}^{\mathrm{e}}$ siècle, de sa signification initiale de "sentinelle postée en un endroit élevé $»^{2}$, le mot passe de façon imagée dans l'expression " mettre en vedette », c'est-à-dire, dans le vocabulaire du théâtre, imprimer, en haut de l'affiche, un nom en caractères plus gros que les autres. L'acteur ainsi mis en valeur devient une « vedette », selon un glissement de sens qui se poursuit lorsque le qualificatif s'applique aussi à un champion réputé meilleur que ses coéquipiers ou ses adversaires : vedettes d'un meeting d'athlétisme

2 Cette acception est encore attestée dans le vocabulaire militaire, puisqu'on continue de placer un soldat « en vedette», dans une fonction de guetteur. 
ou d'un tournoi de tennis, vedettes dans une équipe composée à coups de millions. L'origine du mot - donc du concept - nous l'indique : vedettariat et sport spectacle ont partie liée, pour le meilleur et pour le pire, quand quelque orgueilleux trop vite monté en graine « se prend pour une vedette ».

Puisque ce vedettariat est une question d'affichage, les journalistes en sont les premiers agents, à partir de données objectives : record exceptionnel, extraordinaire moisson de médailles ou exploits individuels réitérés d'un champion transcendant son équipe. La presse, alors, enregistre, relaie et structure la vox populi, qu'en retour elle amplifie. Cependant, en raison du rythme toujours plus accéléré de la communication et de la capacité d'oubli décuplée de lecteurs de moins en moins attentifs à l'histoire, on sait combien, dans un monde où chaque jour davantage une information chasse l'autre, un fait est éphémère, si sensationnel soit-il dans l'instant. Les journaux spécialisés ont beau titrer que tel champion ou telle équipe " entre dans la légende », c'est à peine si l'on s'en souvient un mois plus tard. Il importe donc, pour perpétuer un exploit, une mésaventure injuste illustrant « la glorieuse incertitude du sport » ou la figure emblématique d'une discipline, que d'autres écrits, a priori détachés d'une temporalité présentiste ${ }^{3}$, installent la nouvelle dans la durée (cette dimension est évidemment au cœur du processus ici esquissé). Les vedettes qui résistent à l'estompage de la mémoire et traversent le temps tendent alors réellement à devenir des légendes, en plusieurs étapes.

La première étape consiste en la rédaction d'ouvrages qui mettent en exergue des sportifs, à cette occasion entourés d'un halo romanesque, souvent sur un mode épique ou, au minimum, dramatique : anthologies des grands moments de sport relatés dans la presse spécialisée - le célèbre L'Équipe, cinquante ans de sport (1946-1996) en est sans doute le modèle le plus achevé - et ainsi consacrés par ce seul acte de sélection ; biographies, généralement enjolivées, ou pseudo autobiographies en réalité écrites par des « nègres »; voire récits centrés sur un événement particulier qui,

3 Le terme est ici employé au sens où l'entend François Hartog. 
ipso facto magnifié, prend une valeur au sens propre extraordinaire, tel Le grand combat du quinze de France qui, sous la plume de Denis Lalanne, mythifie la victoire des rugbymen français de 1958 sur ceux de l'Afrique du Sud. Bien qu'ils ne ressortissent pas à la presse, quotidienne ou hebdomadaire, ces textes demeurent pourtant destinés à une consommation presque immédiate. S'ils restent plus longtemps visibles que de simples articles de journal, ils vivent rarement plus que le temps d'une saison et disparaissent aussi vite que les cadeaux de Noël. Quels juniors, même dans un sport comme le rugby qui tire une partie de son identité de la célébration de son histoire, connaissent encore le nom des acteurs de ce « grand combat»?

La deuxième étape nous amène au seuil de la littérature, lorsque les rédacteurs de certains de ces ouvrages font preuve d'un réel talent stylistique, qu'illustrent de façon privilégiée les florilèges d'articles d'Antoine Blondin et les ouvrages de D. Lalanne sur le rugby. Certes, ils ne prétendent pas rompre avec une écriture dont la visée de communication à court terme sature les effets. Par exemple, malgré un titre qui laisse présager un ton épique, Le grand combat du quinze de France de D. Lalanne ne se donne pas pour une épopée. Pour sa part, A. Blondin, pourtant écrivain consacré par l'institution littéraire, ne rassemble certaines de ses contributions à L'Équipe ou à France-Soir dans Ma Vie entre des lignes que pour masquer son impuissance à continuer une création personnelle nettement plus ambitieuse. De même, le prolongement de cette entreprise de florilège rétrospectif, Mes petits papiers, doit sa constitution structurée à un geste post mortem « qui incombe au seul éditeur $\gg$ de cette anthologie, finalement principal responsable d'une « littérarité » acquise a posteriori grâce à une mise en recueil ${ }^{4}$. Mais, si ces auteurs ne s'inscrivent pas explicitement dans le champ de la littérature, ils n'en témoignent pas moins indirectement du processus ici esquissé : ils cherchent à sublimer des exploits ou des champions dans et par le travail d'une écriture. Dans une langue

4 Voir l'avant-propos de Mes petits papiers, proposé par Alain Cresciucci, dont le travail éditorial fait passer en littérature des écrits qui n'avaient pas a priori cette prétention : Crescuicci, 2006, p. 11. C'est à lui que j'emprunte la mise en contexte de Ma Vie entre des lignes. 
qui justement essaie de sortir de la communication ordinaire, ils donnent à lire des faits extraordinaires accomplis par des hommes extraordinaires. Ils essaient de les transformer en légendes.

L'étymologie nous le rappelle, une légende est par essence le produit d'un discours. Le mot latin médiéval legenda, du verbe legere, «lire », désigne en effet, stricto sensu, « ce qui doit être lu», par conséquent ce qui est digne d'être relaté et qu'on fait revivre grâce aux mots qui en perpétuent le souvenir. D'un discours qui exploitait secondairement un spectaculaire préexistant, on passe à un discours en soi créateur d'une dimension supplémentaire : la vedette devient légende. Par conséquent, l'ultime étape du phénomène est son intégration à une œuvre littéraire cette fois revendiquée comme telle. Elle hisse la vedette au-dessus du monde sportif réel pour lui procurer un statut de héros, entendu au sens pleinement romanesque du terme, comme on dit de Cosette ou de Rastignac qu'ils sont des héros.

Telle est mon hypothèse : la vedette du sport se métamorphose en légende du sport si elle est portée par un texte qui dépasse la communication véhiculaire et qui ambitionne au moins de rompre avec «le langage instrument $»^{5}$. La création d'une véritable légende du sport procède d'un saut qualitatif qui, en passant du régime du voir (vedere, vedette) au régime du lire (legere, légende), rompt avec la communication immédiate.

On en trouve un bon exemple dans Courir de Jean Echenoz. Dans ce qui n'est pas une biographie, mais un roman (le terme figure explicitement sur la couverture du livre), Echenoz, écrivain maintes fois consacré par l'institution littéraire, évoque la carrière de Zatopek, coureur tchèque qui s'illustra notamment, sur 5.000 et 10.000 mètres, aux J. O. de 1948 et 1952. Le canal de diffusion de ce texte, «Les Éditions de minuit », véritable estampille, en soi signe et gage de littérarité d'avant-garde, ainsi que la critique journalistique et universitaire qui en a rendu compte, ne laissent aucun doute sur le statut littéraire d'un ouvrage où l'imaginaire d'Echenoz, fasciné par le modèle de la machine ${ }^{6}$, s'accorde

5 Selon la célèbre expression de Sartre dans Qu'est-ce-que la littérature?

6 Voir notamment Le Méridien de Greenwich (1979) et Nous trois (1992). 
particulièrement bien à la célébration de celui qu'on surnomma « la locomotive » parce qu'il imprimait à la course un « train » régulier, inesthétique, mais inexorablement efficace. À partir de l'histoire réelle d'un champion célébré en son temps par les médias et aisément reconnu comme tel par les techniciens et les amateurs un peu soucieux de l'histoire du sport, Zatopek devient l'allégorie de la course de fond, voire de toute course. Comme l'indique le titre du roman, il est devenu la personnification de l'acte même de courir.

Le personnage de Zatopek, jusque dans ses déboires politiques après le printemps de Prague, offre une image sans aspérités, et donc facile à transmuer : une légende, c'est primitivement le récit d'une vie de saint qu'on lisait dans les réfectoires des couvents.

Cette démarche est en revanche moins aisée, et donc plus significative, lorsque la littérature s'empare d'un champion beaucoup plus controversé. C'est pourquoi je testerai mon hypothèse en me penchant sur une figure fascinante mais contestée, Jacques Anquetil. Ce champion d'exception, cependant souvent critiqué pour ses manières hautaines, son goût du lucre et son recours assumé au dopage, est le protagoniste de Anquetil tout seul ${ }^{7}$, écrit par Paul Fournel, éditeur et écrivain consacré, président de l'OuLiPo et auteur de recueils de nouvelles (Les Athlètes dans leur tête reçut dans cette catégorie le prix Goncourt en 1989), de romans et d'essais.

Comment une vedette dont l'image est majoritairement négative et à qui l'imaginaire sportif et populaire français réserve généralement le mauvais rôle dans son emblématique rivalité avec Poulidor, devient-elle une légende positive grâce à la littérature ? Répondre à cette question revient à déployer le titre choisi par Fournel : Anquetil : une vedette insérée dans le monde trouble du cyclisme ; mais en même temps tout seul : une légende hissée au-dessus de l'humaine condition du peloton. Quels sont les processus et les enjeux de ce subtil glissement?

7 Paul Fournel, Anquetil tout seul, Paris, Seuil, 2012. Toutes les références renverront dorénavant à cette édition sous l'abréviation $A$. 


\section{Anquetil...}

Une légende, certes, cultive le merveilleux, mais à partir d'un événement authentique déformé et réinterprété. Il en est ainsi dans Anquetil tout seul qui, comme tout récit, transmet un savoir lié au monde de référence qu'il évoque, celui du cyclisme sur lequel le lecteur trouve au premier degré des informations sociologiques et sportives.

L'arrière-plan réaliste nécessaire à l'évocation du protagoniste fait défiler des compétitions emblématiques (Tour de France, " Grands prix », record de l'heure et critériums ${ }^{8}$ ), des lieux mythiques (Galibier, Puy de Dôme, vélodrome Vigorelli), des champions représentatifs des générations 1950-1960 (Van Looy, Darrigade, et bien sûr Poulidor), voire des précisions très techniques sur les braquets emmenés, les types de vélo utilisés ou les tactiques de course.

À ce décor réaliste, s'ajoute une argumentation implicite, lorsque le narrateur présente un plaidoyer indirect en faveur d'Anquetil sur la question du dopage, évidemment capitale parce qu'elle entre pour beaucoup dans son image négative. Les justifications avancées, destinées à disculper un coureur alors représentatif de sa profession, n'ont rien d'original : le dopage est censé faire partie intégrante du sport de compétition. Anquetil le déclare lui-même : «Il faut être un imbécile ou un faux jeton pour s'imaginer qu'un cycliste professionnel qui court 235 jours par an peut tenir le coup sans stimulants $\|^{9}$. En somme, les cyclistes sont victimes d'un système qui les exploite, mais dont en retour ils tirent profit pour assouvir une volonté respectable et finalement méritoire de s'élever dans la société. Là encore, Anquetil, soucieux de dépasser son statut de petit ouvrier agricole dans l'exploitation familiale, le déclare sans ambages : «Au départ, il y a chez moi un évident désir de promotion sociale ${ }^{10}$. Le voilà, sinon innocenté, du moins largement excusé.

\footnotetext{
8 Par exemple $A$., pp. 34-35, 41-44, 57-59.

9 A., p. 66.

10 A., pp. 56 et 57.
} 
Cependant, ces considérations, qui offrent à l'amateur de sport un premier niveau de lecture, ne s'intègrent pas dans un exposé ordonné explicitement destiné à défendre le coureur. Elles sont disséminées dans un discours littéraire dont elles sont seulement une des cautions réalistes. Dans la mesure où, malgré une mise en œuvre stylistique de premier ordre, elles ne font que reprendre les arguments les plus courants dans le monde du cyclisme, elles ne peuvent à elles seules donner leur patine de héros original à Anquetil, certes champion reposant au Panthéon du vélo, mais alors représentant banal de sa sociologie. Elles sont transcendées par un discours littéraire transmuant Anquetil en une figure légendaire, parce qu'unique.

\section{... Tout seul}

Cette singularité se marque d'abord dans l'ambiguïté générique de la forme littéraire qui la relate et reflète l'ambiguïté existentielle de son protagoniste. Anquetil tout seul se donne explicitement comme un " récit » ${ }^{11}$, c'est-à-dire, au sens strict, une fiction préférant à l'analyse le déroulement de péripéties souvent linéaires et à la première personne ${ }^{12}$. Néanmoins, s'il correspond partiellement à ces caractéristiques, ce « récit », selon une acception moins technique (relation d'une histoire, quelle qu'elle soit), amalgame plusieurs genres volontairement enchevêtrés.

Puisqu'un narrateur extérieur raconte les moments importants de la vie d'Anquetil, le texte ressortit à la biographie. Néanmoins, elle ne suit pas un ordre chronologique strict parce qu'elle est présentée en divers chapitres thématiques inaugurés par un titre (comme « À quoi Anquetil marche-t-il ? »), lui-même suivi de sous-titres (comme « Marche-t-il à l'exploit ? », « Marche-t-il à l'amour du vélo ? », « Marche-t-il à l'argent? ») qui précisent le sujet abordé. Elle est dans cette mesure légèrement contaminée par le genre de l'essai, pourtant finalement refusé, en l'absence de toute argumentation en forme.

11 Le terme figure sur la page de garde après le nom de l'auteur et le titre.

12 Voir Jarrety, 2001, article « Récit ». 
Complexité supplémentaire, sont enchâssés dans la biographie des fragments autobiographiques embrayés, comme au théâtre, par la didascalie « Anquetil ». Les propos rapportés, tantôt repris de vraies déclarations, tantôts inventés, sont alors assumés par un « je » censé être celui du protagoniste.

Mais ils sont à leur tour concurrencés par une autre autobiographie, celle de l'auteur, qui surgit dès le chapitre liminaire en interrompant sans transition une description d'Anquetil ${ }^{13}$, et qui sera ensuite constamment présente. En effet, les deux se superposent : « De 6 à 19 ans, j'ai été Anquetil ${ }^{14}$ ». Ce brouillage déplace les contours du récit, d'autant plus que les rencontres entre les deux héros ne sont pas relatées par ordre chronologique, introduisant un jeu dans une authenticité qu'elles devraient renforcer, mais qui est au contraire de moins en moins assurée au fur et à mesure qu'on remonte dans le temps. Est d'abord relatée la quatrième, authentifiée par une photo, puis la deuxième, simplement virtuelle (« La deuxième fois que j'ai vu Anquetil, je ne l'ai pas vu, je l'ai pisté $\left.»^{15}\right)$, ensuite la troisième, réelle mais réduite à un " instant minuscule » où " Anquetil est passé en un éclair $»^{16}$, et enfin la première, qui n'est que la vision purement imaginaire d'un " fantôme $»^{17}$ : les souvenirs reconstitués par la démarche autobiographique du narrateur jettent sur la biographie d'Anquetil l'ombre portée du fantasme.

Dans et par un genre flou, qui épaissit l'énigme au lieu de la résoudre ${ }^{18}$, est campé un Anquetil rêvé, le mieux à même de réhabiliter un personnage réel trop éclaboussé par une vie un peu scandaleuse. Dès lors se construit la légende, qui hisse Anquetil au-dessus du peloton, groupe compact des coureurs, mais aussi,

13 A. « J'avais 10 ans. J'étais petit, brun et rond... », p. 10.

14 A., p. 144.

15 A., p. 90.

16 A., p. 126.

17 A., p. 148.

18 Voir $A$., pp. 20-21. 
primitivement, amas d'individus indifférenciés fondus dans la masse ${ }^{19}$. « Pour Anquetil, l'essentiel se joue dans la solitude $»^{20}$.

Elle est, certes, d'abord éprouvée en son quotidien de vedette du cyclisme. De son coup de pédale d'une élégance inégalée, Anquetil est irrésistible lors de courses contre la montre justement appelées « effort solitaire » et dans les épreuves en ligne où il dédaigne le commun des " coureurs en troupeau $»^{21}$, au mieux ravalés au rang de domestiques « porteurs d'eau », au pire achetés le temps d'une épreuve.

Mais ce substrat réaliste est d'emblée esthétisé. Dès la première phrase du récit, son protagoniste s'affranchit de sa pesanteur terrestre : " Anquetil jouissait de la bienveillance des vents ». Il sera un peu plus tard question de « grâce », d' « envol » et de « danse $»^{22}$ : Anquetil flotte dans un monde intermédiaire un peu irréel. C'est pourquoi il demeure impénétrable : «Anquetil joue souvent un rôle de personnage mystérieux, et quand il ne le joue pas, il reste un mystérieux personnage $»^{23}$. « Paradoxal $»$, " difficile à comprendre $»^{24}$, il incarne un secret que " lui-même n'est pas très sûr de vouloir connaître " $^{25}$. Il possède « l'âme terrible et la vraie complexité d'un champion » qui précisément ont manqué à Poulidor ${ }^{26}$.

En somme, Anquetil échappe à toute catégorisation. Dès lors qu'on dépasse la simple relation factuelle ou le simple inventaire de ses exploits, on creuse le gouffre de son énigme : « Il est très facile de savoir tout sur Anquetil. En savoir davantage ne fait pourtant qu'épaissir le mystère $»^{27}$. Par conséquent, il faut quitter le terrain plat du reportage pour tenter d'approcher son être profond, c'està-dire en fait son caractère insaisissable. La phrase qui inaugure

19 Voir Rey, 1992, tome 2, p. 1466.

20 A. p. 10.

21 A. p. 32.

22 A. p. 9.

23 A. p. 102.

24 Voir par exemple p. 105, p. 103.

25 A. p. 136.

26 A. p. 88.

27 A. p. 143. 
le deuxième paragraphe de l'ouvrage « son coup de pédale était un mensonge $»^{28}$ n'est pas uniquement la métaphore de l'élégance et de la beauté quasi irréelle d'un cycliste d'exception. Elle évoque un personnage toujours à côté de lui-même, foncièrement problématique comme le sont les meilleurs personnages de roman, contrairement aux vedettes transparentes à elles-mêmes dans l'éclat de leurs succès.

Il n'est donc pas étonnant que la figure d'Anquetil se nimbe de merveilleux, comme il sied à toute légende. Et c'est précisément à l'occasion du Tour de France 1964, lors de la fameuse ascension du Puy de Dôme, sommet de sa rivalité avec Poulidor, que se mesure mieux le phénomène. Cette scène relatée dans toutes les anthologies des exploits cyclistes, et donc profondément inscrite dans la réalité historique du sport, aurait pu faire l'objet d'un décentrement littéraire en tant que réécriture d'un topos épique, présent par exemple dans les westerns ou les chansons de geste, la lutte terminale, au sommet, entre les deux champions de quelque grande cause. Mais Fournel fait bien plus. Il débarrasse Anquetil de son enveloppe matérielle : sur les pentes du Puy de Dôme, Poulidor se

« montrait clairement dans un effort athlétique maximal, à l'extrémité de ses forces et dans la grande clarté de son métier de coureur cycliste. Anquetil, lui, était d'une pâleur de cadavre, les yeux perdus dans un monde secret qui n'était pas celui du vélo, puisant ses forces dans un lieu illisible, dans un puits de mystère $»^{29}$.

L'un est une vedette conceptuellement cernée dans les limites d'une profession, l'autre une légende, qui a quitté le milieu intelligible du cyclisme et même l'univers humain. Il accède au statut de merveille.

Voilà pourquoi cette légende merveilleuse vit non seulement en marge de la réalité (la marginalité est une composante humaine comme une autre), mais contre la réalité. Dans son habituelle

28 A., p. 9.

29 A., p. 44. 
fonction de mise en exergue de l'essentiel de l'ouvrage, le premier épigraphe d'Anquetil tout seul - une citation tirée de The Rider de Tim Krabbé - avait prévenu le lecteur :

« Geldermans me raconta qu'Anquetil, dans les côtes, glissait toujours son bidon dans la poche arrière de son maillot pour alléger son vélo, je décidai d'y regarder de plus près. Je pus constater que, sur toutes les photos d'Anquetil en montagne, son bidon se trouvait bien dans son porte-bidon. Mais c'était une illusion. C'est l'histoire de Geldermans qui disait vrai. C'est celle qui parle au cœur du cycliste. Ce sont les photos qui mentent. »

Seuls les mots peuvent rendre compte d'une légende construite contre la réalité, seule la littérature, même (voire surtout) contre le simple reportage peut transcender la vedette. De fait, il faut finalement prendre au premier degré l'épigraphe qui paraissait n'être qu'une boutade, en effet justifiée par le fragment autobiographique qui, à la fin de l'ouvrage de Fournel, évoque sa première rencontre " pour de vrai $»^{30}$ avec Anquetil, au vélodrome de Saint-Étienne.

«Cette réunion est restée si fort gravée dans ma mémoire de petit garçon que j'en ai mille fois parlé avec mon père, que je l'ai racontée cent fois à mes copains et que j'en ai même composé, trente ans plus tard, une nouvelle dans mon recueil Les Athlètes dans leur tête $»^{31}$.

Mais, cinquante ans après, vérification faite sur des divers documents indiscutables, il se révèle qu'Anquetil n'y était pas.

«L'image d'Anquetil sur la piste en érable du vel'd'Hiv de SaintÉtienne m'a accompagné pendant plus de cinquante ans, elle a fondé ma "passion Anquetil », et ce n'était qu'une image. Dans le baril de son torse, dans les muscles terribles de ses jambes et de son dos, Anquetil cachait ainsi un peu de la fibre dont sont tissés

30 Voir A., p. 145.

31 A., pp. 147-148. 
mes rêves. Peut-être son grand secret était-il, en fin de compte, de se trouver partout où il n'était pas $»^{32}$.

Ainsi, quand le livre se referme, son titre a pris une dimension nouvelle. De l'Anquetil tout seul décrivant sur le mode du « récit» réaliste le champion qui cultivait avec morgue son originalité et le maître des courses contre la montre, on est, dans et par la lecture, insensiblement passé à un Anquetil tout seul évoquant un être unique, comme détaché de l'humaine condition et de tous ses inévitables défauts : une légende.

\section{Conclusion}

Ainsi, quand il est développé par une cuvre littéraire, le discours sur la légende du sport ne repose pas sur la sollicitation d'un ensemble de concepts intégrés dans un raisonnement explicite, ou sur la description euphorisée d'exploits quasiment présentés comme surhumains, comme c'est le cas dans la grande majorité des évocations des « vedettes ».

Il ne vise pas par exemple à réhabiliter Anquetil grâce à des considérations socio-politiques soulignant qu'un fils du peuple a été porté par son désir d'ascension sociale (cause « interne ») et qu'il n'a fait que profiter sans vergogne d'un système presque entièrement organisé à des fins mercantiles (cause « externe »).

L'anti-héros à la fois célébré et vilipendé par la presse se métamorphose en héros légendaire par sa seule saisie dans une forme littéraire, qui opère dans toutes ses dimensions. Au-delà d'un travail stylistique (qu'après tout on trouve aussi chez les meilleurs journalistes), c'est le système porteur de la littérarité qui réalise cette transformation : le choix d'un genre intermédiaire, toujours à cheval entre l'essai thématique, la biographie, l'autobiographie, ainsi que l'installation progressive d'une atmosphère mystérieuse qui " déréalise » Anquetil. Réhabilité dans une fiction qui dépasse et rachète son image ambiguë de vedette controversée, le redoutable homme d'affaires, passé maître dans l'art de gérer 
ses intérêts matériels, devient un être immatériel hors du monde intelligible et donc aussi hors du temps. Dès lors, inévitablement « tout seul», Anquetil se trouve, selon les derniers mots du livre, « partout où il n’était pas ». La littérature le fige en légende.

\section{Références}

Crescuicci, A. (2006). (éditeur de Mes petits papiers d'Antoine Blondin). Paris : La Table ronde.

Echenoz, J. (2009). Courir. Paris : Les Éditions de Minuit.

Fournel, P. (2012). Anquetil tout seul. Paris : Seuil.

Hartog, Fr. (2002). Régimes d'historicité. Présentisme et expériences du temps. Paris : Seuil.

Jarrety, M. (2001). Lexique des termes littéraires. Paris : Livre de poche.

Rey, A. (1992). Dictionnaire historique de la langue française. Paris : Le Robert.

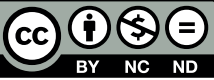
«Attribution - Pas d'Utilisation Commerciale - Pas de Modification 4.0 International» (CC BY-NC-ND 